\title{
Associação entre a produção anual de ouro em garimpos e incidência de malária em Mato Grosso - Brasil, 1985-1996
}

\author{
Association between reported annual gold mining extraction and \\ incidence of malaria in Mato Grosso-Brazil, 1985-1996
}

\author{
Elisabeth Carmen Duarte ${ }^{1,2,3}$ e Cor Jesus Fernandes Fontes ${ }^{3,4}$
}

\begin{abstract}
Resumo Baseado em dados secundários realizou-se análise epidemiológica do tipo ecológico da série histórica da incidência parasitária anual da malária, produção oficial anual garimpeira de ouro e gastos financeiros do Programa de Controle da Malária na Bacia Amazônica relativo ao Estado de Mato Grosso, no período de 1985-1996. Associação positiva e estatisticamente significante $(p<0,001)$ entre produção de ouro e IPA foi observada em análise multivariada, ainda que controlada por gastos financeiros. Esse achado contribui para a elucidação da tendência de redução da malária em MT, observada na última década.
\end{abstract}

Palavras-chaves: Malária. Garimpos. Controle de endemias.

Abstract A secondary data analysis was performed using an ecological design to study the association between malaria incidence rates, the reported annual production of gold mining extraction and monetary investments for the control of malaria from 1985 to 1996 in Mato Grosso, Brazil. A positive and statistically significant $(p<0.001)$ association between the amount of gold extracted and MIR was obtained in multivariate regression analysis, even after allowing for financial investments in malaria control activities. This finding contributes to an understanding of the decrease observed in malaria incidence in Mato Grosso during the last decade, in view of the significant decrease in gold mining within the region during this period.

Key-words: Malaria. Gold mining. Disease control.

A extração manual de ouro no Brasil (garimpo) gera reconhecido impacto ambiental, mobilização rápida e volumosa de grupos populacionais, com rápido crescimento demográfico e súbitas mudanças na estrutura socioeconômica das populações ${ }^{2}$. Aliado a isso, as populações-alvo das atividades garimpeiras na Amazônia brasileira apresentam características peculiares, que complicam ainda mais ações interventoras, tais como: 1) estão localizadas em áreas pouco desenvolvidas e de acesso difícil; 2) vivem em ambiente físico altamente favorecedor à proliferação de doenças transmitidas por vetores, em especial a malária; 3) são compostas principalmente por migrantes sem qualquer organização profissional; 4) afetam de maneira direta e indireta o modo de vida das populações autóctones ${ }^{1}$. Como conseqüência observa-se deterioração dos serviços públicos de saúde, podendo chegar ao colapso e grande incompatibilidade para o atendimento quantitativo e qualitativo das demandas geradas nesse processo.
Em Mato Grosso (MT), desde o período colonial, a mineração tem representado uma atividade produtiva responsável pela expansão e criação de muitos núcleos urbanos ${ }^{9}$. Porém, foi na década de 70 que inúmeros focos de descoberta de ouro foram instalados neste estado. Em 1984, 13 grandes empresas do ramo da mineração encontravam-se em atividade no Estado, sendo que no ano de 1988 a produção industrial de ouro em MT atingiu seu pico de produção. Porém, a partir do ano de 1991, grande decréscimo dessa produção é observado. A queda da produção de ouro no estado de MT pode ser explicada por vários fatores, entre eles $^{9}$ : a exaustão dos depósitos aluvio-coluvionares (ouro de superfície), o que passa a requerer domínio de técnicas de engenharia mais sofisticadas para a exploração do minério em profundidades maiores, elevando o custo de sua extração. Paralelamente, nesse momento (1992), o preço internacional do ouro encontrava-se relativamente baixo (inferior a US $\$ 400$ a onça troy) tornando pouco vantajosa economicamente a extração do minério.

\footnotetext{
1. Fundação Nacional de Saúde (FUNASA), Cuiabá, MT. 2. Centro de Pós-Graduação da Universidade de Cuiabá (UNIC). 3. Núcleo de Estudos de Doenças Infecciosas e Tropicais de Mato Grosso, Cuiabá, MT. 4. Universidade Federal de Mato Grosso, Cuiabá, MT, Brasil.

Endereço para correspondência: Dr. Cor Jesus Fernandes Fontes, R. Wenceslau Bras 355, Morada do Sol, 78043-508 Cuiabá, MT, Brasil Telefax: $5565621-1406$

E-mail: fontes@terra.com.br

Recebido para publicação em 19/2/2002.
} 
Associadas a esses fatos, ações de interdição de garimpos em MT coincidiram com a descoberta de vários outros garimpos no Estado do Pará, localizado mais ao norte da Região Amazônica. Resultado disso foi a evasão de grande parte da população garimpeira do norte de Mato Grosso, confirmada pelas contagens da população feitas nos anos de 1991 e $1996^{34}$.

Concomitantemente, o índice parasitário anual (razão do número de exames positivos de malária pela população) no Estado de MT apresentou declínio importante, principalmente a partir de 1992, assim como a mortalidade por esta causa básica ${ }^{81011}$. As razões desta queda têm sido discutidas pelos profissionais do setor saúde, e constituem em objetivo desta análise.

Baseado em dados secundários realizou-se análise epidemiológica do tipo ecológico da série histórica da incidência parasitária anual da malária (IPA), produção oficial anual garimpeira de ouro (produção de ouro), e gastos financeiros do Programa de Controle da Malária na Bacia Amazônica (PCMAM) relativos ao MT (gastos financeiros do PCMAM), do período de 1985 a 1996. As fontes desses dados foram: Fundação Nacional de Saúde de Mato Grosso 7810 11; Departamento Nacional da Produção Mineral $^{3}$ e relatório técnico do Programa de Controle da Malária na Bacia Amazônica ${ }^{5}$. A unidade de análise foram os anos estudados de 1985 a 1996 ( $n=12)$, a variável dependente foi a IPA registrada nesses anos e as variáveis independentes foram a produção de ouro e os gastos financeiros do PCMAM. A produção de ouro foi utilizada como marcadora da exposição da população aos riscos da malária devido (direta ou indiretamente) a essa atividade ocupacional. Outros gastos de rotina com o programa de controle da malária (não oriundos do PCMAM) foram considerados constantes no período.

Em 1985 a IPA da malária para o Estado de MT foi de 8,3 exames positivos por 1.000 habitantes, a qual apresentou ascensão constante anual até 1992, quando observou-se IPA de 95,2 por 1.000 habitantes $^{78}$. A partir de 1992, no entanto, um declínio progressivo deste indicador foi observado, atingindo em 1995 IPA de 25,5 por 1.000 habitantes $^{810}$. Considerando a população de 2.235.832 habitantes em $\mathrm{MT}^{4}$, constataram-se os seguintes indicadores epidemiológicos da malária em 1996: índice anual de exames de sangue para malária (IAES) de 6,9 para cada 100 habitantes e IPA de 16,83 para cada 1.000 habitantes (Tabela 1). Essa redução da IPA foi reproduzida em virtualmente todos os municípios de maior transmissão no Estado.

\begin{tabular}{|c|c|c|c|}
\hline Ano & IPA (x1000 hab) & Gastos (xUS\$1.000) & Produção de ouro $(\mathrm{kg})$ \\
\hline 1985 & 8,3 & 0 & 5.142 \\
\hline 1986 & 11,7 & 0 & 2.828 \\
\hline 1987 & 12,7 & 0 & 4.556 \\
\hline 1988 & 15,3 & 0 & 5.175 \\
\hline 1989 & 31,2 & 1.895 & 4.557 \\
\hline 1990 & 73,4 & 1.738 & 25.231 \\
\hline 1991 & 71,8 & 515 & 27.053 \\
\hline 1992 & 95,2 & 221 & 22.211 \\
\hline 1993 & 59,5 & 893 & 16.285 \\
\hline 1994 & 44,1 & 2.676 & 13.153 \\
\hline 1995 & 25,5 & 5.647 & 8.471 \\
\hline 1996 & 16,8 & 0 & 6.945 \\
\hline
\end{tabular}

Fonte: Referências 6, 7, 8, 9

Paralelamente, observaram-se mudanças importantes nos investimentos no programa de controle da malária a nível nacional, destacando-se como mais importantes os investimentos originários do PCMAM. Segundo relatório de avaliação ${ }^{5}$, os gastos financeiros do PCMAM destinados ao controle da malária no Estado de MT, originalmente nulos, atingiram, em 1989 e 1990, valores de US\$1.895.000 e US\$1.738.000 respectivamente. Posteriormente, houve queda desses valores nos anos seguintes, tendo sido reduzido a US\$221.000,00 em 1992. A partir deste ano (1992), porém, observou-se grande investimento no setor, com gastos chegando ao volume de US\$5.647.000,00 no ano de $1995^{5}$ (Tabela 1).
Nesse mesmo momento histórico, no entanto, observou-se um importante fenômeno na produção aurífera do Estado. A produção oficial garimpeira de ouro, que no final da década de 80 era da ordem de $5.000 \mathrm{~kg}$ extraídos anualmente, chegou a atingir $27.053 \mathrm{~kg} \mathrm{em}$ 1991. A partir daí, no entanto, essa produção apresentou marcado declínio, atingindo em 1996 apenas 6.945kg extraídos ${ }^{9}$ (Tabela 1).

Em análise da associação dessas séries históricas, observaram-se os seguintes resultados: para o período em que havia algum financiamento do PCMAM, 1989 a $1995(n=7)$, uma associação negativa e estatisticamente significante entre gastos financeiros do PCMAM e IPA é observada em análise de regressão linear univariada 
$(\beta=-0,011, I C 95 \%=-0,020$ a $-0,0009 ; p=0,037)$. Esta associação, no entanto, perde significância estatística $(p=0,170)$ quando controlada pela produção de ouro no Estado. Por outro lado, uma associação positiva e estatisticamente significante é observada entre produção de ouro e IPA $(\mathrm{p}=0,05)$, mesmo após ser controlada por gastos financeiros.

Resultados semelhantes são observados se for considerado o período completo de análise (1985 a 1996, $\mathrm{n}=12$ ) (Tabela 2). Com as variáveis gastos financeiros do PCMAM e produção de ouro no modelo de regressão, observou-se que produção de ouro apresenta associação estatisticamente significante com a variação da IPA, o que não acontece com os gastos financeiros do PCMAM (Figura 1). Este modelo prediz uma aumento médio da IPA (x 1.000 habitantes) em $0,31($ IC95\%=0,22-0,40) para cada incremento de $100 \mathrm{~kg}$ de ouro extraídos no Estado anualmente (F-teste parcial $=29,16, p=0,0001$ ), ainda que controlando os gastos financeiros do PCMAM. Não foi identificada interação entre os efeitos das duas variáveis independentes analisadas e IPA $(p=0,75)$. O resultado da regressão linear estimada encontra-se na Tabela 2.

Esses achados indicam forte associação entre a atividade garimpeira (mensurada pela produção de ouro) e a incidência da malária no estado de Mato

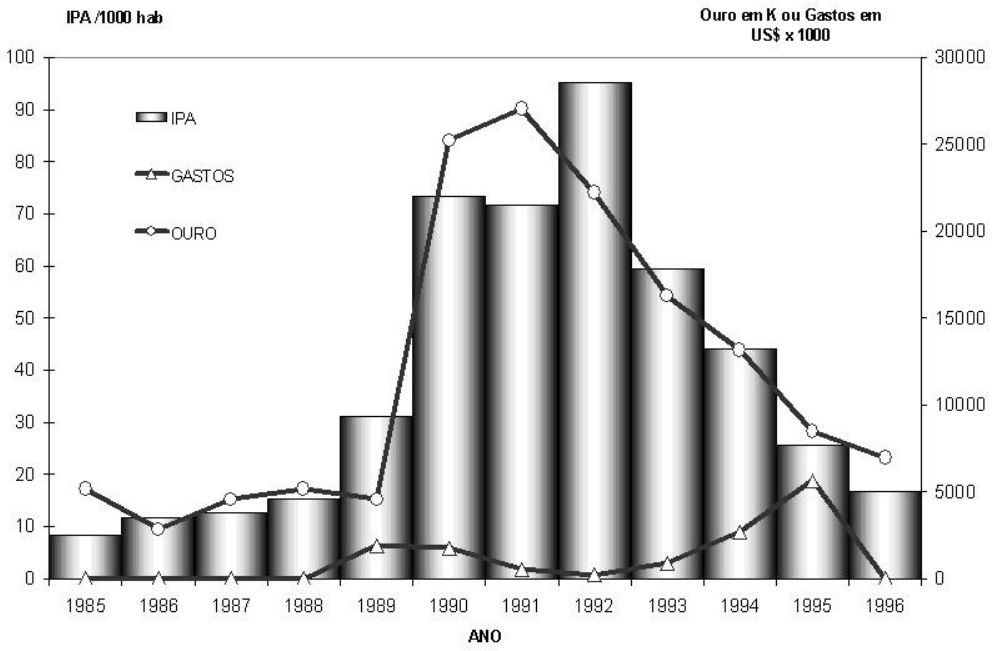

Figura 1 - Incidência parasitária anual (IPA), gastos financeiros do programa de controle da malária na bacia amazônica (PCMAM) e produção garimpeira de ouro em Mato Grosso, Brasil, 1985-1996.

Tabela 2 - Resultados da análise de regressão linear da associação entre produção de ouro e gastos financeiros do PCMAN* com IPA* de malária no período de 1985 a 1996 em Mato Grosso (Brasil).

\begin{tabular}{lrr}
\hline Variável & Coeficiente $(\beta)$ & Valor de $p$ \\
\hline Produção de ouro $(\mathrm{kg})$ & 0,0031 & $<0,001$ \\
Gastos financeiros do PCMAN (US\$1.000,00) & 0,0002 & 0,912 \\
Constante & 1,899 & - \\
\hline$R^{2}=0,837$, F-teste $\mathrm{p}=0,0001$ & &
\end{tabular}

* PCMAN = Programa de controle da malária na bacia amazônica; IPA = Incidência parasitária anual de malária.

Grosso, ainda que controlados os gastos financeiros destinados ao controle da doença. Explicação para esse fato pode ser encontrada na hipótese de que a queda da produção aurífera tenha determinado evasão maciça de parcela de uma população especialmente sob alto risco de transmissão da malária na região e/ou de mudança de sua atividade ocupacional, o que também ocasionaria decréscimo de risco de transmissão da malária nesses grupos populacionais. Por outro lado, não se afirma aqui que o investimento financeiro no controle da malária não tenha tido impacto em sua transmissão. No entanto, nesse momento em que a determinação da doença parece estar tão fortemente vinculada a um fenômeno tão impactante como a redução drástica dos garimpos e da exposição ambiental das populações, o impacto da intensificação das medidas de controle, em conseqüência a um maior investimento financeiro, não pode ser mensurado.

Por outro lado, limitações desse tipo de análise podem ter gerado vieses nos resultados, afetando a validade das conclusões aqui apresentadas. Isso pode ser verdade principalmente se as variáveis de grupos 
populacionais (ecológicas) aqui analisadas não se aplicarem aos indivíduos e se variáveis de confusão importantes tenham sido omitidas da análise, tais como mudanças climáticas, mudanças em aspectos da organização social desses grupos populacionais e da oferta de serviços de saúde, entre outras. Além disto, a análise realizada assume independência das observações, segundo unidades de análise (anos), independência essa que possivelmente tenha sido violada $^{6}$. Apesar da hipótese aqui apresentada para a explicação da redução da malária em Mato Grosso parecer encontrar sustentabilidade nesses resultados, devido às limitações inerentes à análise epidemiológica utilizada, ela deve ser equacionada em estudos de avaliação metodologicamente mais robustos.

\section{REFERÊNCIAS BIBLIOGRÁFICAS}

1. Camara VM, Corey G. Epidemiologia e meio ambiente: o caso dos garimpos de ouro no Brasil. Centro Panamericano de Ecologia Humana e Saúde, Organização Panamericana de Saúde/Organização Mundial de Saúde, México, 1992.

2. Farid LH. Diagnóstico preliminar dos impactos ambientais gerados por garimpos de ouro em Alta Floresta - MT: um estudo de caso. CETEM/CNPq, Rio de janeiro, 1992.

3. Fundação Instituto Brasileiro de Geografia e Estatística. Censo demográfico de 1991 - resultados preliminares. IBGE, Rio de Janeiro, 1992

4. Fundação Instituto Brasileiro de Geografia e Estatística. Contagem da população 1996. IBGE, Rio de Janeiro, 1997.

5. Fundação Nacional de Saúde. Avaliação final do Programa de Controle das Doenças Endêmicas do Nordeste e Programa de Controle da Malária na Bacia Amazônica. FNS, Brasília, 1996.

6. Kleinbaum DG, Kupper LL, Muller KE. Applied regression analysis and other multivariable methods. PWS-KENT, Boston, 1988.
7. Marques AC. Dados epidemiológicos da malária na Amazônia, por município, referentes a 1992. Revista da Sociedade Brasileira de Medicina Tropical 26:43-59, 1993.

8. Marques AC. Dados epidemiológicos de malária em todo o Brasil, referentes a 1993. Revista da Sociedade Brasileira de Medicina Tropical 28:141-155, 1995.

9. Miranda JG, Cipriani M, Martires RAC, Giaconi WJ. Atividades garimpeiras no Brasil: aspectos técnicos, econômicos e sociais. CETEM/CNPq, Rio de Janeiro, 1997.

10. Passos AD, Fialho RR. Malária, aspectos epidemiológicos e de controle. Revista da Sociedade Brasileira de Medicina Tropical 31(supl 2):93-105, 1998.

11. Superintendência de Campanhas de Saúde Pública. Dados epidemiológicos da Malária no Brasil referentes ao período de 1985 a 1989. Revista da Sociedade Brasileira de Medicina Tropical 23: 55-62, 1990. 